\title{
DYNAMIC VS. STATIC MAINTENANCE RATE POLICIES FOR MULTI-STATE QUEUEING SYSTEMS
}

\author{
${\text { Gang } \text { Chen }^{1, *} \text {, Zaiming Liu }}^{2}$ And YuQing Chu ${ }^{3}$
}

\begin{abstract}
This paper considers a single-server queueing system with server breakdowns. When the server fails, it is sent to repair immediately and its maintenance rate is allowed to be adjustable. The goal is to find the optimal maintenance rates that minimize the long-run average cost of the system. We address the static and dynamic maintenance control problems respectively. For the static control model, we derive the stationary system performances and the explicit solution of the optimal maintenance rate. Then regarding the dynamic maintenance problem, we formulate it as a Markov decision process (MDP) and the optimal dynamic policy is proved to be a threshold policy. Based on the structure of optimal dynamic policy, we construct a performance evaluation for computing efficiently the optimal threshold and average cost. Finally, a comparative study of the dynamic and static policies is presented by numerical experiment which shows the impact of system parameters on the optimal maintenance policies.
\end{abstract}

Mathematics Subject Classification. 90B22, 60K25.

Received October 23, 2019. Accepted April 3, 2020.

\section{INTRODUCTION}

Due to wear, erosion and other causes, the unreliable machine is typically subject to unpredictable breakdowns. When such an event occurs, the failed machine will be sent to repair by allocation of human resources, which is one of the most important factors for decreasing the total cost of the system [9]. Meanwhile the operation will be interrupted until the breakdown is repaired. In order to reduce the loss of demands, it is essential for the manager to take the optimal maintenance policy in both service and manufacturing firms in the sense that the performance measures and optimization are of primary concerns $[17,23]$.

In this paper we study the optimal static and dynamic maintenance problems in queueing systems where the server with two working states may be subject to failures at random. When the server breaks down, the new arrivals will be lost until it restarts. The breakdowns of server will result in a temporarily unavailable period depending on varying maintenance rates, and therefore the performances of the systems will be heavily affected. The decision variable is the maintenance rate of the failed server. The objective is find the optimal maintenance policy to minimise a average cost (including the lost, holding and maintenance costs) over an infinite

Keywords. Queueing system, maintenance rate, MDP, performance evaluation.

1 Business School, Sun Yat-sen University, Guangzhou 510275, P.R. China.

2 School of Mathematics and Statistics, Central South University, Changsha 410083, P.R. China.

3 School of Science, Wuhan University of Technology, Wuhan 430070, P.R. China.

* Corresponding author: chengmathcsu@163.com 
planning horizon. This study of the multi-state queueing system with server breakdowns is motivated by its wide applications [3,22]. For example, in the automated car wash facility situation, as the facility operates, it may gradually deteriorate due to wear and it will fall into a subnormal state with a higher breakdown probability or lower service rate. The repair time of the failed machine mainly depends on the repair techniques and resources, which can be described by the maintenance rate in the view of the mathematical model. Due to the fact that more repair resources will lead to smaller repair time and less lost, but greater repair cost. So that for this tradeoff, it is important to take optimal maintenance rate (MR) policy so as to minimize the average cost of the system. Our proposed model is suitable for this situation where a customer is typically a car waiting for being washed and the automated car wash facility can be regarded as a server with two working states which differ in their breakdown rates. To the best of our knowledge, there is no research work on the proposed model. With this article, we aim to contribute to this discussion by providing insights on how optimal MR policy can be taken into account in queues with server breakdowns and the potential benefits of dynamic policy over static policy on system performances. The article does not attempt to develop a decision support tool that can readily be used in real time. Our goal, rather, is to develop a relatively simple model that captures the static and dynamic MR policies, and provide some guidance to the situations mentioned in this paper.

Our key results in this paper are the following: First, the optimization problems are formulated based on queueing systems with two working states of the server. Second, we derive the stationary queue-size distributions in the static and dynamic systems, respectively. Third, we give the explicit expressions for the solution of the static maintenance problem. Meanwhile, we analyse the structural properties of the optimal dynamic maintenance policy and use the performance evaluation to efficiently compute it. Finally, we also present a sensitivity analysis for several system parameters and the benefit of the dynamic policy by numerical experiments. To the best of our knowledge, investigating the static and dynamic MR problems simultaneously and their comparison in the stochastic systems are not addressed. The benefits of dynamic maintenance policy with respect to static maintenance policy are not always obvious. This study will fill this gap and it could provide manager theoretic and feasible decision information of the maintenance policy.

The remainder of this paper is structured as follows. In Section 2 we present a literature review on maintenance policy and models. We describe the mathematical model and the optimization problems in Section 3. For the static maintenance problem, we obtain the steady state distribution of the system and the closed form of the optimal static policy in Section 4. In Section 5, the dynamic maintenance problem is formulated as a Markov decision problem. We derive the structure of the optimal policy and average cost by a performance evaluation model. In Section 6, the sensitivity analysis of the system parameters and the benefit of the dynamic policy are illustrated by numerical experiments. Finally, some further discussions and conclusions are provided in Section 7 .

\section{Literature OVERVIEW}

Optimization problem is a focal topic in the research of maintenance theory. A mount of research papers with respect to the maintenance policies in queueing systems have been published in the past decades. In the study of maintenance theory, various maintenance policies under different scenarios that may model some real applications have been investigated. In recent years, most articles analyzed the emergency maintenance $(\mathrm{EM})$, preventive maintenance $(\mathrm{PM})$ and corrective maintenance $(\mathrm{CM})$ policies $[4,16,21]$. While, the modeling of maintenance rate policy in queueing systems are surprisingly limited. In this paper, we study the optimal static and dynamic MR policy in queues. Our paper is closely related to two streams of literature, one dealing with static maintenance policy and the other with dynamic maintenance policy in queues.

The first stream is on static maintenance control of queueing systems. For the static maintenance problem, the methodology that most articles use is that of mean value analysis. Kouedeu et al. [8] considered control of production and corrective maintenance rates in a system with multiple-machine. They answered the question that if the contribution of the approach of controlling the machines repair rates in terms of total cost reduction is significant compared to a fixed repair rate situation. Kenne and Nkeungoue [7] studied the control problem of $\mathrm{CM}$ and $\mathrm{PM}$ rates in a manufacturing system where the decision variables are the preventive maintenance 
rate of the machine and its repair rate. Wang et al. [19] investigated the M/M/1 machine repair problem with working vacation in which the server works with different repair rates rather than completely terminating the repair during a vacation period. Wu et al. [20] dealt with an multi-server queueing system where the servers are assumed unreliable and a controllable repair policy is introduced. A cost model is constructed to determine the optimal value of repair rate. Gao et al. [6] studied a repairable discrete-time Geo/G/1 queueing system. They derived the system performances and a cost function is analyzed by taking the repair rate as a decision variable.

The second stream is on dynamic maintenance control of queueing systems. For the dynamic maintenance problem, Kim and Makis [10] considered optimal maintenance policy for a multi-state deteriorating system with two types of failures. A modified policy-iteration algorithm using the embedded technique is developed as the computational approach used to find the optimal maintenance policy. Abeygunawardane et al. [1] studied the adaptive equipment's maintenance schedules policy for aging devices. They found optimal maintenance policies for their proposed MDP model by the backward induction algorithm. Borrero [2] derived the structural properties of the inventory dependent optimal PM policy by using MDP approach. Njike et al. [14] investigated the simultaneous control of maintenance and production rates of a manufacturing system with defective products. They considered the partial stochastic model with constant demand rates and exponential failure and repair times distributions of the machines. They investigated the joint control policies and show that the optimal maintenance policy has a bang bang structure (i.e., the hedging point policy is optimal) by numerical experiment.

In our paper, we assume that all the processes in our model are stochastic. Moreover, we derive the similar result with [14] for the structure of the optimal maintenance policy and provide its rigorous proof by MDP theory instead of their analysis by numerical techniques. This paper not only analyzes the characterization of the optimal MR policy, but also proposes an efficient algorithm for optimal dynamic policy based on a performance evaluation method. In addition, almost all the papers quoted above just consider onefold control policy (static or dynamic). Few papers have studied the benefit of dynamic maintenance policy with respect to the static policy.

\section{Model Formulation}

We consider a standard FCFS unreliable queueing system with Poisson arrivals at rate $\lambda$ and independent exponential services with rate $\mu$. In this model, the working server has two states (normal state and sub-normal state) which differ in their breakdown probabilities $\alpha_{0}$ and $\alpha_{1}\left(\alpha_{0}<\alpha_{1}\right)$. When the server breaks down, the customer arrivals are not allowed to join the queue. The failed server can be repaired to be normal state after an exponentially distributed time with rate $\gamma$. The time duration of the normal state until the sub-normal state is exponentially distributed with rate $\beta$. As noted by $[5,12]$, the assumption of exponential times is reasonable in many service systems and has only a limited impact on the model precision. The system state at time $t$ can be described by $X(t)=\{(L(t), J(t)), t \geq 0\}$ where $L(t)$ and $J(t)$ represent the number of customers in the system (including the customer in service) and the server state at time $t$ respectively. The states of the server are as follows

$$
J(t)= \begin{cases}0, & \text { normal state } \\ 1, & \text { sub-normal state } \\ 2, & \text { maintenance state }\end{cases}
$$

In this section, we present the static and dynamic maintenance problems, respectively. We formulate the dynamic maintenance problem as a continuous-time review model where maintenance decisions can be made at any point of time. The static maintenance problem formulation is identical except that a unique maintenance rate has to be chosen for the whole time horizon.

\subsection{Dynamic maintenance problem}

In this model, we first consider the state-dependent maintenance rate problem. We assume that the set of allowable maintenance rates $\gamma$ is a bounded interval of the form $[\underline{\gamma}, \bar{\gamma}]$. The unit variable maintenance cost is 
TABLE 1. Main notations.

\begin{tabular}{ll}
\hline \hline Symbol & Description \\
\hline $1 / \lambda$ & Expected inter-arrival time of the customers \\
$1 / \mu$ & Expected service time of each customer \\
$1 / \gamma$ & Expected maintenance time of the failed server \\
$1 / \beta$ & Expected time from normal to sub-normal state of the server \\
$\alpha_{0}\left(\alpha_{1}\right)$ & Breakdown probability of the normal (sub-normal) server \\
$\bar{\gamma}(\underline{\gamma})$ & Maximum (minimal) maintenance rates \\
$h$ & Holding cost per unit time for each customer in the system \\
$c$ & Maintenance cost rate for the failed server per unit time \\
$r$ & Cost for each lost customer \\
\hline
\end{tabular}

$c$ and each lost customer due to the failed server will occur a cost $r$. Each customers in the system will occur a holding cost $h$. We summarize now the previous notations (Tab. 1).

For the dynamic maintenance problem, the manager chooses a maintenance rate from a compact set $[\gamma, \bar{\gamma}]$ at any decision epoch. The set of decision epochs is composed of the set of the times when the system state changes. Our objective is to obtain the optimal dynamic MR policy to minimize the average cost. The expected discounted cost (the sum of holding, lost and maintenance costs) over a finite time $T$ under a policy $\pi$ and an initial state $(i, j)$ can be written as:

$$
v_{T, \alpha}^{\pi}(i, j)=E_{(i, j)}^{\pi}\left[\int_{0}^{T} e^{-\alpha t} h L(t) \mathrm{dt}+\int_{0}^{T} e^{-\alpha t} r d M(t)+\int_{0}^{T} e^{-\alpha t} c \gamma(t) \mathrm{dt}\right],
$$

where $M(t)$ denotes the number of the customers that have lost up to time $t$ and $\gamma(t)$ represents the maintenance rate used at time $t$, and $\alpha$ is the discount rate.

We consider the stationary Markov policy $\pi$ under which the system evolves as a continuous-time Markov chain. Due to the Markovian property, the optimal policy depends only on the current state of the system. As it is known from Tijms [18], the long-run average cost $g_{d}(\pi)$ for the policy $\pi$ can be written in the following form,

$$
g_{d}(\pi)=\lim _{T \rightarrow \infty} v_{T}^{\pi}(i, j) / T
$$

in which $v_{T}^{\pi}(i, j)$ denotes the total expected costs up to time $T$ when the system starts in state $(i, j)$ under a policy $\pi$, i.e., $v_{T}^{\pi}(i, j)=\lim _{\alpha \rightarrow 0} v_{T, \alpha}^{\pi}(i, j)$. The goal of the manager is to find an optimal dynamic policy $\pi^{*}$ that minimizes the long-run average cost:

$$
g_{d}=\min _{\pi \in \Pi} g_{d}(\pi)=g_{d}\left(\pi^{*}\right),
$$

where $\Pi$ is the set of all admissible dynamic MR policies. Using the standard tools of uniformization and normalization, we cast the problem as a Markov decision problem and construct a discrete-time equivalent of our original queueing system. Without loss of generality, we assume that $\lambda+\mu+\bar{\gamma}+\beta=1$. Now we consider a relative real-valued function $v(i, j)$ which is defined on the state space. The relative value function can be regarded as the asymptotic difference in total costs that results from starting the process in state $(i, j)$ instead of some reference state. As is shown in Puterman [15], the optimal policy $\pi^{*}$ and average cost $g_{d}$ are the solutions of the optimality equation

$$
T_{m} v(i, j)=v(i, j)+g_{d},
$$


where $T_{m}$ is the dynamic programming operator acting on $v$, defined as follows

$$
\begin{aligned}
& T_{m} v(i, 0)=\left\{\begin{array}{cc}
\lambda v(i+1,0)+\beta v(i, 1)+\left(1-\alpha_{0}\right) \mu v(i-1,0) & i \geq 1 \\
+\alpha_{0} \mu v(i-1,2)+\delta v(i, 0)+i h & i=0, \\
\lambda v(1,0)+\beta v(0,1)+(1-\lambda-\beta) v(0,0) &
\end{array}\right. \\
& T_{m} v(i, 1)=\left\{\begin{array}{cc}
\lambda v(i+1,1)+\alpha_{1} \mu v(i-1,2)+\left(1-\alpha_{1}\right) \mu v(i-1,1) & i \geq 1 \\
+(1-\lambda-\mu) v(i, 1)+i h & i=0, \\
\lambda v(1,1)+(1-\lambda) v(0,1) &
\end{array}\right. \\
& T_{m} v(i, 2)=\min _{\gamma \in[\underline{\gamma}, \bar{\gamma}]}\{\gamma v(i, 0)+(1-\gamma) v(i, 2)+c \gamma\}+i h,
\end{aligned}
$$

in which $\delta=1-\lambda-\beta-\mu$.

\subsection{Static maintenance problem}

The setting of the static maintenance problem is similar, except that the maintenance rate cannot be changed over time. Let us consider first the problem with a given maintenance rate $\gamma$. The problem is then to find the optimal maintenance rate policy minimizing the average cost over an infinite horizon. Based on the cost elements, we develop the expected average cost function $g_{s}(\gamma)$ for the queueing system with static MR policy as follows,

$$
g_{s}(\gamma)=h E[L]+r E[N]+c \gamma
$$

where $E[N]$ denotes the expected lost customers per unit time, and $E[L]$ represents the expected number of customers in the system under a fixed maintenance rate $\gamma$. Following the construction of the expected average cost function, our objective is to determine the optimum value of the control parameter $\gamma$, say $\gamma^{*}$, so as to minimize the expected average cost. The static maintenance problem can be formulated as

$$
g_{s}^{*}=\min _{\gamma \in[\underline{\gamma}, \bar{\gamma}]} g_{s}(\gamma)
$$

\section{Characterization of the optimal static policy}

In this section, we focus on the structure of the optimal static maintenance policy. The problem can be analyzed by using a technique known as mean value analysis. It requires a clearly defined static server discipline and the performance measures of the system. To do so, we first analyze the the stationary performances of the system. For the system with a fixed maintenance rate $\gamma$, it is clear that the process $X(t)$ is a two-dimensional continuous time Markov chain with state space $E=\{(i, j) \mid i=0,1,2 \ldots ; j=0,1,2\}$ and its transition rate diagram is given in Figure 1.

Denote by $\pi_{i, j},(i, j) \in E$ the steady-state distribution of the Markov chain $X(t)$. The corresponding stationary distribution is obtained as the unique positive normalized solution of the following balance equations:

$$
\begin{aligned}
(\lambda+\beta) \pi_{0,0} & =\left(1-\alpha_{0}\right) \mu \pi_{1,0}+\gamma \pi_{0,2}, \\
\lambda \pi_{0,1} & =\left(1-\alpha_{1}\right) \mu \pi_{1,1}+\beta \pi_{0,0}, \\
\gamma \pi_{i, 2} & =\alpha_{0} \mu \pi_{i+1,0}+\alpha_{1} \mu \pi_{i+1,1}, \\
(\lambda+\beta+\mu) \pi_{i+1,0} & =\lambda \pi_{i, 0}+\left(1-\alpha_{0}\right) \mu \pi_{i+2,0}+\gamma \pi_{i+1,2}, \\
(\lambda+\mu) \pi_{i+1,1} & =\lambda \pi_{i, 1}+\left(1-\alpha_{1}\right) \mu \pi_{i+2,1}+\beta \pi_{i+1,0},
\end{aligned}
$$




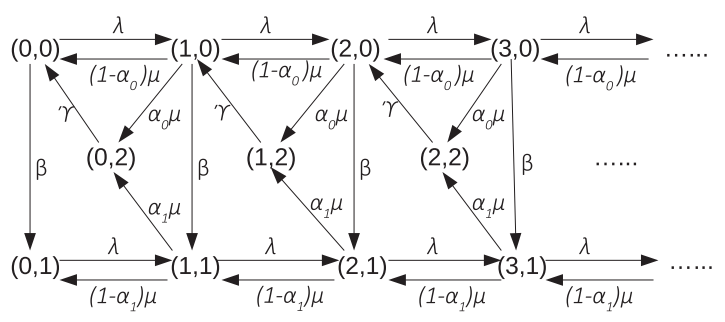

Figure 1. Transition rate diagram of the system under the static MR policy.

where $i \geq 0$. In order to derive an analytic solution for the steady-state probability $\pi_{i, j}$ in closed form expression, probability generating function (PGF) techniques must be used since solving equations (4.1)-(4.5) using a recursive method is difficult. We define the following PGFs:

$$
p_{k}(z)=\sum_{i=0}^{\infty} \pi_{i, k} z^{i}, \quad k=0,1,2 .
$$

Next we investigate the stationary queue-size distribution, which enables us to obtain closed form expressions for various performance measures. The main results of the distribution of steady state are given in the following theorem and its proof can be found in Appendix A.1.

Theorem 4.1. If the stability condition $\rho=\lambda / \mu<1$ holds, then the stationary probabilities of the system states are given by:

$$
\pi_{n, 0}=\pi_{0,0} \rho^{n}, \quad \pi_{n, 1}=\pi_{0,1} \rho^{n}, \quad \pi_{n, 2}=\left(\beta+\lambda \alpha_{0}\right) \pi_{0,0} \rho^{n} / \gamma .
$$

The stationary distribution of the number of customers in the system is geometric distribution: $\pi_{n}=(1-\rho) \rho^{n}$ in which $\pi_{0,0}$ and $\pi_{0,1}$ are given by (A.10).

Let $p_{0}, p_{1}$ and $p_{2}$ be the probabilities of the server on normal, sub-normal and maintenance states respectively. Taking $z=1$ into equation (A.11), we have,

$$
p_{k}=p_{k}(1)=\frac{\mu \pi_{0 k}}{\mu-\lambda}, \quad k=0,1, \quad p_{2}=p_{2}(1)=\frac{\mu\left(\alpha_{0} \lambda+\beta\right) \pi_{00}}{\gamma(\mu-\lambda)} .
$$

Let $L_{0}, L_{1}$ and $L_{2}$ be the expected number of customers in the system when the server is in normal, sub-normal and maintenance states respectively. Then we have

$$
L_{0}=p_{0}^{\prime}(1)=\frac{\lambda \mu \pi_{00}}{(\mu-\lambda)^{2}}, \quad L_{1}=p_{1}^{\prime}(1)=\frac{\lambda \mu \pi_{01}}{(\mu-\lambda)^{2}}, \quad L_{2}=p_{2}^{\prime}(1)=\frac{\lambda \mu\left(\beta+\lambda \alpha_{0}\right) \pi_{00}}{\gamma(\mu-\lambda)^{2}} .
$$

Once the PGFs of the system states are calculated, we can obtain some important performance measures of the system. To simplify the presentation, we define $\rho=\lambda / \mu$ and summary the main results of the performance measures as follows.

(1) The expected number of costumers in the system is given by

$$
E[L]=L_{0}+L_{1}+L_{2}=\frac{\left[\lambda \mu \gamma \alpha_{1}+\mu \beta \gamma+\lambda \mu \alpha_{1}\left(\beta+\lambda \alpha_{0}\right)\right] \pi_{00}}{\alpha_{1} \gamma(\mu-\lambda)^{2}}=\frac{\rho}{1-\rho} .
$$

(2) The expected sojourn time for a customer in the system is given by

$$
E[W]=\frac{E[L]}{\lambda}=\frac{1}{\mu-\lambda} .
$$


(3) The expected number of lost customers per unit time is given by

$$
E[N]=\lambda p_{2}=\frac{\lambda \mu\left(\alpha_{0} \lambda+\beta\right) \pi_{00}}{\gamma(\mu-\lambda)}
$$

For equation (3.1) and the performance measures obtained above, the expected cost function $g_{s}(\gamma)$ per unit time in the static model is given by

$$
g_{s}(\gamma)=\frac{h \rho}{1-\rho}+r \frac{\left(\alpha_{0} \lambda+\beta\right) \lambda^{2} \alpha_{1}}{\gamma\left(\alpha_{1} \lambda+\beta\right)+\left(\alpha_{0} \lambda+\beta\right) \lambda \alpha_{1}}+c \gamma
$$

Since the structure of the function $g_{s}(\gamma)$ is complex and non-linear in terms of $\gamma$, we consider the convexity (unimodal) property by its first-order and second-order. We present the optimal solution $\gamma^{*}$ in the following theorem and its proof is provided in Appendix A.2.

Theorem 4.2. In the static control model, the optimal maintenance rate $\gamma^{*}$ is given as follows:

$$
\gamma^{*}=\left\{\begin{array}{lll}
\underline{\gamma} & \text { if } \quad \gamma^{\prime}<\underline{\gamma} \\
\gamma^{\prime} & \text { if } \quad \underline{\gamma} \leq \gamma^{\prime} \leq \bar{\gamma} \\
\bar{\gamma} & \text { if } \quad \gamma^{\prime}>\bar{\gamma}
\end{array}\right.
$$

where $\gamma^{\prime}=\sqrt{\frac{r\left(\alpha_{0} \lambda+\beta\right) \lambda^{2} \alpha_{1}}{c\left(\alpha_{1} \lambda+\beta\right)}}-\frac{\left(\alpha_{0} \lambda+\beta\right) \lambda \alpha_{1}}{\alpha_{1} \lambda+\beta}$.

\section{Characterization of the optimal Dynamic Policy}

Now, we focus on deriving the properties of the optimal dynamic policy $\pi^{*}$ which will provide basal insight for us to find the optimal policy with less computational effort due to a reduction of the solution search space [11]. We characterise the structure of the optimal policy by investigating the properties of the relative value function and optimal equation. To do so, we first show that the relative value function $v(i, j)$ satisfies the property as specified in the following lemma of which the proof is given in Appendix A.3.

Lemma 5.1. In the dynamic control model, the optimal relative value function $v(i, j)$ satisfies: $v(i, 0)-v(i, 2) \geq$ $v(i+1,0)-v(i+1,2)$ for all $i \geq 0$.

Denote $\Delta v(i)=v(i, 0)-v(i, 2)$ and Lemma 5.1 shows that $\Delta v(i)$ is non-increasing with respect to $i$. Now, we can get the properties of the optimal policy by this property of the relative value function. The proof of the following theorem can be found in Appendix A.4.

Theorem 5.2. In the dynamic control model, the optimal policy is a bang-bang control policy with a threshold $N^{*}$, i.e., $\gamma^{*}=\underline{\gamma}$ if $i<N^{*}$, otherwise $\gamma^{*}=\bar{\gamma}$. The threshold is defined as follows:

$$
N^{*}=\min \{i: v(i, 0)-v(i, 2)+c \leq 0\} .
$$

This theorem show the characteristic of the optimal dynamic policy. While in order to implement this principe, one needs to then answer the following question: How to compute the optimal threshold and the average cost. In remainder of this section, we present some results aiming to solve it. Based on the structure of the optimal policy, we formulate a performance evaluation model for computing efficiently the optimal threshold $N^{*}$ and average cost. Different from the value iteration algorithm which depends on the values of the system parameters and require truncation of the state space, the performance evaluation method which makes best of the characteristic of the optimal policy is more efficiently for the threshold type policy model.

The approach that we take follows from the recognition that a system operating under a control policy specified by a fixed threshold $N$ can be modeled as a Markov chain. In particular, the system state in our 


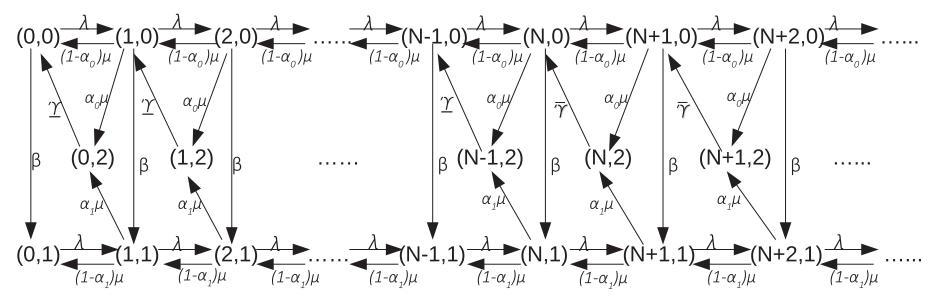

Figure 2. Transition rate diagram of the system under the threshold policy.

model $X(t)=\{(L(t), J(t)), t \geq 0\}$ with the state space $E=\{(i, j) \mid i=0,1,2, \ldots, j=0,1,2\}$ evolves as a twodimension continuous time Markov chain for any choice of threshold $N$. And its corresponding transition rate diagram is presented in Figure 2. Then the infinitesimal generator $Q$ of this system is given by

$$
Q=\left(\begin{array}{ccccc}
A_{00} & B & & & \\
C & A_{11} & B & & \\
& C & A_{22} & B & \\
& & \ddots & \ddots & \ddots
\end{array}\right)
$$

where the matrices $B, C$ and $A_{i j}, i, j \geq 0$ are square matrices of order 3 , which are represented by,

$$
B=\left(\begin{array}{ccc}
\lambda & 0 & 0 \\
0 & \lambda & 0 \\
0 & 0 & 0
\end{array}\right), \quad C=\left(\begin{array}{ccc}
\left(1-\alpha_{0}\right) \mu & 0 & \alpha_{0} \mu \\
0 & \left(1-\alpha_{1}\right) \mu & \alpha_{1} \mu \\
0 & 0 & 0
\end{array}\right), \quad A_{00}=\left(\begin{array}{ccc}
-\lambda-\beta & \beta & 0 \\
0 & -\lambda & 0 \\
\underline{\gamma} & 0 & -\underline{\gamma}
\end{array}\right),
$$

and if $1 \leq i \leq N-1$,

$$
A_{i i}=\left(\begin{array}{ccc}
-\lambda-\beta-\mu & \beta & 0 \\
0 & -\lambda-\mu & 0 \\
\underline{\gamma} & 0 & -\underline{\gamma}
\end{array}\right)
$$

If $i \geq N$,

$$
A_{i i}=\left(\begin{array}{ccc}
-\lambda-\beta-\mu & \beta & 0 \\
0 & -\lambda-\mu & 0 \\
\bar{\gamma} & 0 & -\bar{\gamma}
\end{array}\right) .
$$

As is shown above, our model has the standard structure of a QBD process with infinitesimal generator $Q$. We could use the matrix-geometric solution. Here, we briefly outline the computation of the steady state probability. Let $\Pi=\left(\Pi_{0}, \Pi_{1}, \Pi_{2}, \ldots\right)$ denotes the steady-state probability vector of the generator $Q$. Under the stability condition $\rho<1$, the stationary distribution of the Markov chain $\{(L(t), J(t)), t \geq 0\}$ is always exist. We take the efficient method developed by Latouche and Ramaswami [13] to compute the stationary distribution. To this end, first we denote an auxiliary matrix $Q(R)$ as follows.

$$
Q(R)=\left(\begin{array}{cccccc}
A_{00} & B & & & & \\
C & A_{11} & B & & & \\
& C & A_{22} & B & & \\
& & \ddots & \ddots & \ddots & B \\
& & & C & A_{N-1 N-1} & B C+A_{N N}
\end{array}\right)
$$

where $R$ is a rate matrix which can be determined by the quadratic equation $B+C R+A_{N N} R^{2}=0$. Based on the infinitesimal generator $Q$, we can derive the stationary probabilities of the system as specified in the following theorem, the proof of which can refer to [13]. 
Theorem 5.3. The stationary probabilities of the system states satisfy the relationship:

$$
\Pi_{\mathbf{k}}=\Pi_{\mathbf{N}} R^{k-N}, \quad k \geq N,
$$

in which $\Pi_{\mathbf{0}}, \Pi_{\mathbf{1}}, \ldots, \Pi_{\mathbf{N}}$ are determined by the equations

$$
\left(\Pi_{\mathbf{0}}, \Pi_{\mathbf{1}}, \ldots, \Pi_{\mathbf{N}}\right) Q(R)=0, \quad \sum_{k=0}^{N-1} \Pi_{\mathbf{k}} \mathbf{e}+\Pi_{\mathbf{N}}(I-R)^{-1} \mathbf{e}=1 .
$$

It is obviously that under the stability condition $\rho<1$, the two-dimensional stochastic process $X(t)=\{(L(t), J(t)), t \geq 0\}$ is an ergodic continuous time Markov chain for any fixed threshold $N$. As it is known from Tijms [18], the long-run average cost of the system under the threshold policy $N$ can be written as the following form,

$$
g_{d}(N)=\lim _{T \rightarrow \infty} \frac{v_{T}(i, j)}{T}=h \sum_{i=1}^{\infty} \sum_{j=0}^{2} i \pi_{i j}+r \lambda \sum_{i=0}^{\infty} \pi_{i 2}+c \underline{\gamma} \sum_{i=0}^{N} \pi_{i 2}+c \bar{\gamma} \sum_{i=N+1}^{\infty} \pi_{i 2},
$$

where $\pi_{i j}$ is the steady-state probability of the state $(i, j) \in E$. Therefore once the stationary probabilities are calculated, the long-run average cost per unit time under any fixed threshold policy $N$ can be obtained. So that the optimal dynamic control problem can be formulated as the following programming problem:

$$
\text { Objective: } g_{d}^{*}=\min g_{d}(N) \text { subject to }: N \in\{1,2,3, \ldots\} \text {. }
$$

The minimum average cost $g_{d}^{*}$ can be computed efficiently and the optimal dynamic policy parameter $N^{*}$ can be obtained via an exhaustive search over a large enough range of $N$. Though the search space is very high, we take the Newton-type optimization method which can save a lot of numerical computation power. Compared with the Howard's iteration algorithm, the computational effort for carrying out this search is generally modest and convenient. This approach makes full use of the structure of optimal policy.

\section{Numerical Results}

In this section, we provide numerical results on the optimal policies and average costs obtained in Sections 4 and 5 for the static and dynamic models. In Section 6.1 we take a closer look at the effects of the system parameters on the optimal policy and average cost. In Section 6.2 we investigate the benefit of dynamic policy on average costs by comparing with the static policy. Moreover, considering the relative implement cost of the static and dynamic policies, we proposed a numerical analysis method to provide the manager more decision information. Here we selected the following examples because they demonstrate the most general structure for the optimal static and dynamic MR policies that we observed from several numerical examples.

\subsection{Effects of parameters on optimal policy and average cost}

In this subsection, we briefly provide some numerical examples that examine the sensitivity of the optimal policy and average cost to some system parameters. For each numerical experiment, we compute the optimal values of the policy and average cost. The numerical results show the impact of system parameters $\lambda, \mu$ and $\beta$ on the optimal policy parameters $\gamma^{*}, N^{*}$ and average costs $g_{s}^{*}, g_{d}^{*}$ in corresponding models. The following arbitrary values of system parameters are assumed as follows $\gamma \in[0.1,0.6], \alpha_{0}=0.1, \alpha_{1}=0.3, h=2, r=10, c=5$. As is shown in the tables, we can make the following observations.

In Tables 2-4, we describe the characteristics of the policy parameters $\gamma^{*}, N^{*}$ and average costs $g_{s}^{*}, g_{d}^{*}$ in association with $\lambda, \mu$ and $\beta$. As we can see from Tables $2-4$, the average costs $g_{s}^{*}$ and $g_{d}^{*}$ increase as $\lambda$ and $\beta$ increase, while decreases as $\mu$ increases. For the optimal threshold, we observe that as the system parameters $\lambda$ and $\beta$ increase, the policy parameter $N^{*}$ increases, while decreases as $\mu$ increases, which show a staircase-like 
TABLE 2. Optimal control parameters and costs vs. $\lambda$ for $\mu=1, \beta=0.1$.

\begin{tabular}{|c|c|c|c|c|}
\hline \multirow{2}{*}{ Arrive rate $\lambda$} & \multicolumn{2}{|c|}{ Static policy model } & \multicolumn{2}{|c|}{ Dynamic policy model } \\
\hline & Parameter $\gamma^{*}$ & Average cost $g_{s}^{*}$ & Parameter $N^{*}$ & Average cost $g_{d}^{*}$ \\
\hline 0.60 & 0.249 & 5.9990 & 7 & 5.1934 \\
\hline 0.62 & 0.257 & 6.3508 & 7 & 5.6192 \\
\hline 0.64 & 0.265 & 6.7316 & 7 & 6.2886 \\
\hline 0.66 & 0.272 & 7.1465 & 8 & 6.8285 \\
\hline 0.68 & 0.281 & 7.6020 & 8 & 7.4532 \\
\hline 0.70 & 0.287 & 8.1062 & 8 & 7.9980 \\
\hline 0.72 & 0.295 & 8.6696 & 9 & 8.5649 \\
\hline 0.74 & 0.302 & 9.3061 & 9 & 9.2072 \\
\hline 0.76 & 0.310 & 10.0339 & 9 & 9.9601 \\
\hline 0.78 & 0.317 & 10.8781 & 10 & 10.8203 \\
\hline 0.80 & 0.325 & 11.8735 & 10 & 11.8100 \\
\hline
\end{tabular}

TABLE 3. Optimal control parameters and costs vs. $\mu$ for $\lambda=0.7, \beta=0.2$.

\begin{tabular}{lllllc}
\hline \hline \multirow{2}{*}{ Service rate $\mu$} & \multicolumn{2}{c}{ Static policy model } & & \multicolumn{2}{c}{ Dynamic policy model } \\
\cline { 2 - 3 } \cline { 5 - 6 } & Parameter $\gamma^{*}$ & Average cost $g_{s}^{*}$ & & Parameter $N^{*}$ & Average cost $g_{d}^{*}$ \\
\hline 0.80 & 0.303 & 17.7086 & 12 & 17.0510 \\
0.82 & 0.303 & 15.3753 & & 11 & 14.6801 \\
0.84 & 0.303 & 13.7086 & & 11 & 13.0513 \\
0.86 & 0.303 & 12.4586 & & 10 & 11.8200 \\
0.88 & 0.303 & 11.4864 & & 10 & 9.8112 \\
0.90 & 0.303 & 10.7086 & & 9 & 8.7802 \\
0.92 & 0.303 & 10.0723 & & 9 & 7.8227 \\
0.94 & 0.303 & 9.5420 & & 8 & 6.1101 \\
0.96 & 0.303 & 9.0923 & & 8 & 5.5064 \\
0.98 & 0.303 & 8.7086 & & & 5.9540 \\
1.00 & 0.303 & 8.3753 & & & \\
\hline
\end{tabular}

TABLe 4. Optimal control parameters and costs vs. $\beta$ for $\lambda=0.8, \mu=1$.

\begin{tabular}{llllll}
\hline \hline \multirow{2}{*}{ Fall rate $\beta$} & \multicolumn{2}{c}{ Static policy model } & & \multicolumn{2}{c}{ Dynamic policy model } \\
\cline { 2 - 3 } \cline { 5 - 6 } \cline { 5 - 6 } & Parameter $\gamma^{*}$ & Average cost $g_{s}^{*}$ & & Parameter $N^{*}$ & Average cost $g_{d}^{*}$ \\
\hline 0.10 & 0.325 & 11.8735 & 6 & 10.8951 \\
0.12 & 0.330 & 11.9521 & & 6 & 11.0270 \\
0.14 & 0.334 & 12.0203 & & 7 & 11.2272 \\
0.16 & 0.337 & 12.0815 & & 7 & 11.4101 \\
0.18 & 0.340 & 12.1327 & & 7 & 11.6321 \\
0.20 & 0.343 & 12.1793 & 8 & 11.7902 \\
0.22 & 0.345 & 12.2217 & 8 & 11.9125 \\
0.24 & 0.347 & 12.2596 & 8 & 12.0512 \\
0.26 & 0.349 & 12.2940 & 8 & 12.1305 \\
0.28 & 0.350 & 12.3253 & 8 & \\
0.30 & 0.352 & 12.3538 & 9 & \\
\hline
\end{tabular}



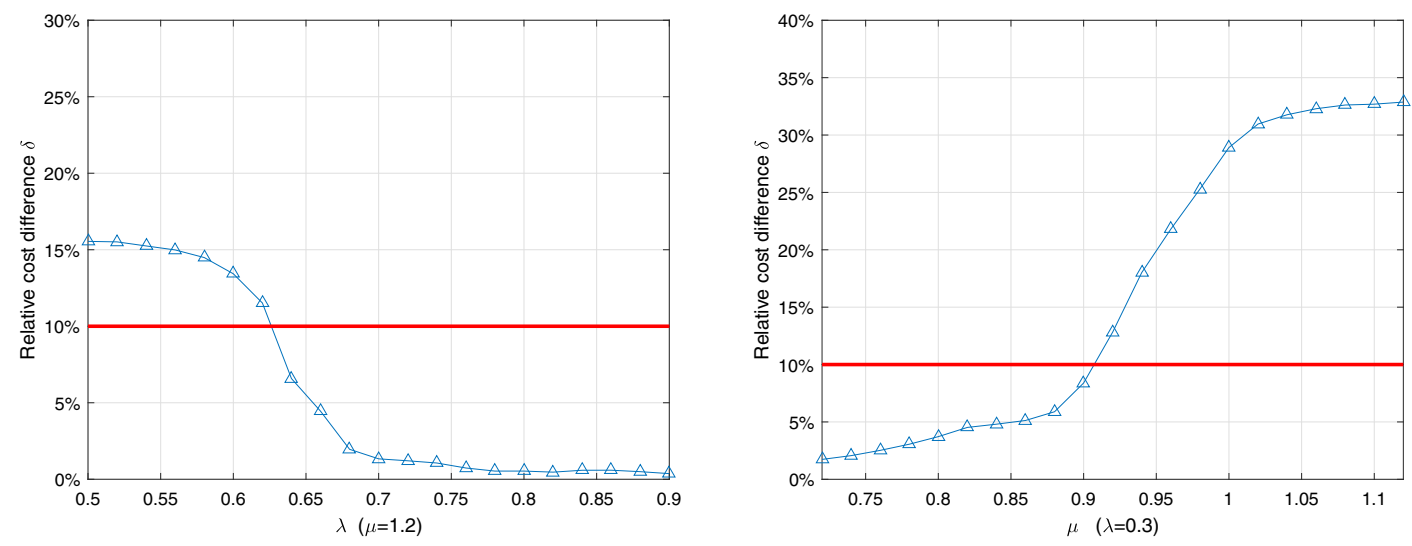

FiguRE 3. Relative cost differences when using static policy instead of dynamic policy $(h=2, c=5)$.

increasing or decreasing pattern. Furthermore, as $\lambda$ and $\beta$ increase, the optimal maintenance rate $\gamma^{*}$ increases. While Table 4 shows that the optimal maintenance rate $\gamma^{*}$ remains constant with $\mu$ increasing. This is expected because the parameter $\gamma^{\prime}$ is independent of $\mu$. It is noted in these tables that $g_{d}^{*}<g_{s}^{*}$, which indicates that the dynamic policy makes much better on minimizing the average cost.

\subsection{The benefit of the optimal dynamic policy}

In this subsection, we study the benefit of the dynamic policy by making a comparative analysis of the average costs under the static and dynamic policies. To do so, the relative cost difference is defined by the relative average cost increase by $\delta=\left(g_{s}^{*}-g_{d}^{*}\right) / g_{s}^{*}$ that results from using static policy instead of the dynamic policy. For the view of the system average cost, it is clear that the dynamic policy works much better than the static policy. While as we all know, since the dynamic control policy needs the manager to monitor the number of the customers in the system, it is more complex to carry out in the practical application. It would reproduce somehow the complexity of the optimal policy and would be relatively difficult to implement in practice. Moreover, the time to find the optimal policy parameters is exponential within the number of policy parameters.

Considering these factors, here we define the relative implement cost of the dynamic policy by a scale $K$, which can be evaluated by the cost elements in different systems. In other word, the manager in the system should focus on the tradeoff between the relative cost difference of the system $\delta$ and the relative implement cost of the dynamic policy $K$. Therefore in term of the overall situation (average cost of the system and the implement of the policy), there is a decision principle that the manager will choose the static policy if $\delta<K$, otherwise for the dynamic policy. Next, some numerical examples are provided to illustrate the effectiveness of the proposed analysis methods in different kind of cases. We set the parameters as follows $\gamma \in[0.1,0.6]$, $\beta=0.1, \alpha_{0}=0.1, \alpha_{1}=0.3, r=10$, and $K=10 \%$ which is labeled with red line in the following figures.

From Figure 3, we present results that show the sensitivity of the relative cost difference $\delta$ to the system parameters $\lambda$ and $\mu$. We find that the relative cost difference $\delta$ decreases as the parameter $\lambda$ increases, while increases as the parameter $\mu$ increases. We observe that the relative cost differences $\delta$ in Figure 3 are among a large range and the lines of the relative cost differences cross the level of $K=10 \%$. That is, within certain ranges of the system parameters, the dynamic policy is not always much better than the static policy. Figure 4 shows respectively the effect of $h$ and $c$ on the relative cost difference $\delta$. As the holding cost parameter $h$ increases, the relative cost difference $\delta$ decreases, while the parameter $c$ has the opposite impact on the relative cost difference $\delta$. In addition, we find that when the parameter $c$ changes within certain ranges, the relative cost difference $\delta$ is always smaller than the value of $K=10 \%$. 

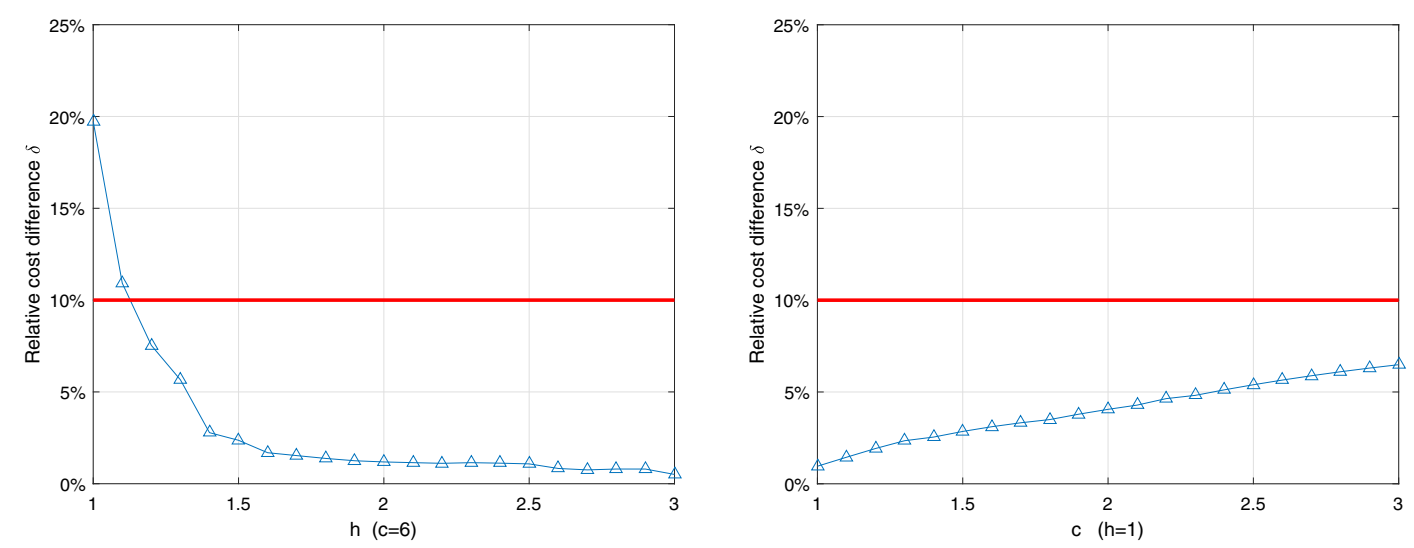

Figure 4. Relative cost differences when using static policy instead of dynamic policy $(\lambda=0.8, \mu=1)$.

Furthermore, all of the figures show that the relative cost differences $\delta$ in the present numerical examples are among a large range $1.01 \%-33.6 \%$. This result indicates that the benefit of dynamic policy heavily depends on the system parameters, so that it is necessary for the manager to consider the system parameters before making a decision. For these experiments if the relative implement cost scale $K$ satisfies $K<1.01 \%(K>33.6 \%)$, it is better for the manager to take the dynamic (static) policy for these cases, otherwise the manager should use the different policy types based on the vary system parameters. Therefore it is clear that the manager should consider the system parameters to determine whether takes the dynamic policy or not. In a word, for the optimization of MR control problem in the unreliable system, the manager can conduct some numerical comparisons of the optimal average costs under static policy and dynamic policy by the method proposed in the present paper. Then the manager takes the relative implement cost of the dynamic policy into consideration to decide which policy to take.

\section{CONCLUSiOn}

This paper considered the optimal control policy in an queueing system with server breakdowns. In order to minimize the expected average cost, the manager takes the MR policy to deal with the tradeoff including the holding cost, maintenance cost and lost cost. Our goal is to find the optimal MR policy under two settings: the static and dynamic maintenance policies. We obtained the closed-form of the optimal static policy and show that the optimal dynamic control policy is of threshold type. For the dynamic control model, we constructed a performance evaluation model for computing the optimal threshold and average cost. Furthermore, we analyzed the behaviour of optimal parameters and average cost with varying system parameters. A comparison of the dynamic policy and static policy was presented by numerical experiments.

It is worthwhile to note that the proposed method in this paper can be applied to the class of models. In this paper, we consider the unreliable model with one server and homogeneous customers. One possible change is to study the model with multi-server or heterogeneous customers which we will study in the near future. Another way to generalize the model is to apply the embedded Markov chain and semi-Markov decision processes to consider the system with general service time or maintenance time, which is also a interesting topic for future research. 


\section{Appendix A.}

\section{A.1. Proof of Theorem 4.1}

From equations (4.1) and (4.4), (4.1) is multiplied by $z^{0},(4.4)$ by $z^{i+1}(i \geq 0)$, and the equations are added term by term for all possible of all values of $i$, we finally get

$$
(\lambda+\mu+\beta) p_{0}(z)-\mu \pi_{00}=\lambda z p_{0}(z)+\frac{\left(1-\alpha_{0}\right) \mu\left[p_{0}(z)-\pi_{00}\right]}{z}+\gamma p_{2}(z) .
$$

Similarly, for equations (4.2), (4.3) and (4.5), we have

$$
\begin{gathered}
\gamma p_{2}(z)=\frac{\alpha_{0} \mu\left[p_{0}(z)-\pi_{00}\right]}{z}+\frac{\alpha_{1} \mu\left[p_{1}(z)-\pi_{01}\right]}{z} \\
(\lambda+\mu) p_{1}(z)-\mu \pi_{01}=z \lambda p_{1}(z)+\frac{\left(1-\alpha_{1}\right) \mu\left[p_{1}(z)-\pi_{01}\right]}{z}+\beta p_{0}(z) .
\end{gathered}
$$

Solving equations (A.1) to (A.3), we obtain

$$
\begin{aligned}
& p_{2}(z)=\frac{A(z) p_{0}(z)+\left(1-\alpha_{0}\right) \mu \pi_{00}-z \mu \pi_{00}}{\gamma z}, \\
& p_{1}(z)=\frac{\left(z-1+\alpha_{1}\right) \mu \pi_{01}+\beta z p_{0}(z)}{B(z)} \\
& p_{0}(z)=\frac{\mu\left[B(z) \pi_{00}+\alpha_{1} \lambda z \pi_{01}\right]}{(\mu-\lambda z)[B(z)+z \beta]}
\end{aligned}
$$

in which $A(z)=(\lambda+\beta+\mu) z-\lambda z^{2}-\left(1-\alpha_{0}\right) \mu$ and $B(z)=(\lambda+\mu) z-\lambda z^{2}-\left(1-\alpha_{1}\right) \mu$. Then, the generating function of the queueing length can be expressed by

$$
L(z)=p_{0}(z)+p_{1}(z)+p_{2}(z) .
$$

Substituting equations (A.4) to (A.6) into equation (A.7), we get

$$
\begin{aligned}
L(z)= & \frac{B(z)\left[z B(z) \mu \gamma+z^{2} \beta \mu \gamma+A(z) B(z) \mu+\left(1-\alpha_{0}-z\right) \mu f(z)\right] \pi_{00}}{B(z) f(z) \gamma z} \\
& +\frac{\left[z^{2} B(z) \lambda \mu \gamma \alpha_{1}+z^{3} \gamma \lambda \mu \beta \alpha_{1}+z f(z) \gamma \mu\left(z-1+\alpha_{1}\right)+z B(z) A(z) \lambda \mu \alpha_{1}\right] \pi_{01}}{B(z) f(z) \gamma z},
\end{aligned}
$$

where $f(z)=(\mu-\lambda z)[B(z)+\beta z]$. This expression contains all known quantities, except for two unknown probabilities $\pi_{0,0}$ and $\pi_{0,1}$ which can also be determined.

One linear equation can be obtained by invoking the normalizing condition that $L(1)=1$. Another linear equation can be derived by complex analysis. By Rouche's theorem, we get that the denominator of the above equation has exactly only one solution (assume $z=z_{0}$ ) satisfying $B\left(z_{0}\right)+\beta z_{0}=0$ inside the closed unit disk of the complex z-plane except for $z=0$, so that the numerator of the above equation equals zero when $z=z_{0}$. Hence, we have

$$
\begin{aligned}
\beta \pi_{0,0}-\alpha_{1} \lambda \pi_{0,1} & =0, \\
\mu\left[\gamma G_{1}+\mu \alpha_{1} G_{0}-\alpha_{0} f(1)\right] \pi_{0,0}+\left[\gamma \lambda G_{1}+\mu \lambda \alpha_{1} G_{0}+\gamma f(1)\right] \pi_{0,1} & =\gamma f(1),
\end{aligned}
$$

in which $G_{0}=\mu \alpha_{0}+\beta$ and $G_{1}=\mu \alpha_{1}+\beta$. It follows from equations (A.8) and (A.9) that

$$
\begin{aligned}
\pi_{0,0} & =\frac{\gamma \lambda(\mu-\lambda) \alpha_{1}}{\gamma \mu\left(\alpha_{1} \lambda+\beta\right)+\alpha_{1} \lambda \mu\left(\alpha_{0} \lambda+\beta\right)}, \\
\pi_{0,1} & =\frac{\beta \gamma(\mu-\lambda)}{\gamma \mu\left(\alpha_{1} \lambda+\beta\right)+\alpha_{1} \lambda \mu\left(\alpha_{0} \lambda+\beta\right)} .
\end{aligned}
$$


After manipulating equations (A.4) to (A.6) and (A.10), we have

$$
p_{k}(z)=\frac{\mu \pi_{0 k}}{\mu-\lambda z}, \quad k=0,1, \quad p_{2}(z)=\frac{\mu\left(\alpha_{0} \lambda+\beta\right) \pi_{00}}{\gamma(\mu-\lambda z)} .
$$

It follows from above equations and the definition of PGF that

$$
\frac{\mu \pi_{00}}{\mu-\lambda z}=\sum_{n=0} \pi_{0,0} \rho^{n} z^{n}, \quad \frac{\mu \pi_{01}}{\mu-\lambda z}=\sum_{n=0} \pi_{0,1} \rho^{n} z^{n}, \quad \frac{\mu\left(\alpha_{0} \lambda+\beta\right)}{\gamma(\mu-\lambda z)}=\frac{\beta+\lambda \alpha_{0}}{\gamma} \sum_{n=0} \pi_{0,0} \rho^{n} z^{n},
$$

which lead to

$$
\pi_{n, 0}=\pi_{0,0} \rho^{n}, \quad \pi_{n, 1}=\pi_{0,1} \rho^{n}, \quad \pi_{n, 2}=\left(\beta+\lambda \alpha_{0}\right) \pi_{0,0} \rho^{n} / \gamma .
$$

So that we obtain $\pi_{n}=\pi_{n, 0}+\pi_{n, 1}+\pi_{n, 2}=(1-\rho) \rho^{n}$. This completes the proof.

\section{A.2. Proof of Theorem 4.2}

From equation (4.6), we know the expression of expected cost function $g_{s}(\gamma)$ in the static model. Taking the first order and second order of $g_{s}(\gamma)$ with respect to $\gamma$, we have

$$
\begin{aligned}
g_{s}^{\prime}(\gamma) & =c-\frac{r\left(\alpha_{0} \lambda+\beta\right)\left(\alpha_{1} \lambda+\beta\right) \lambda^{2} \alpha_{1}}{\left[\gamma\left(\alpha_{1} \lambda+\beta\right)+\left(\alpha_{0} \lambda+\beta\right) \lambda \alpha_{1}\right]^{2}}, \\
g_{s}^{\prime \prime}(\gamma) & =\frac{r\left(\alpha_{0} \lambda+\beta\right)\left(\alpha_{1} \lambda+\beta\right)^{2} \lambda^{2} \alpha_{1}}{\left[\gamma\left(\alpha_{1} \lambda+\beta\right)+\left(\alpha_{0} \lambda+\beta\right) \lambda \alpha_{1}\right]^{3}} .
\end{aligned}
$$

It is obvious that $g_{s}^{\prime \prime}(\gamma)>0$ which means that the function $g_{s}(\gamma)$ is convex with respect to $\gamma$. Since $\gamma \in[\underline{\gamma}, \bar{\gamma}]$, we only have to solve $g_{s}^{\prime}(\gamma)=0$ to find the feasible solutions of $\gamma^{\prime}$ in $[\underline{\gamma}, \bar{\gamma}]$. By solving the equation $g_{s}^{\prime}(\gamma)=0$, we have

$$
\gamma^{\prime}=\sqrt{\frac{r\left(\alpha_{0} \lambda+\beta\right) \lambda^{2} \alpha_{1}}{c\left(\alpha_{1} \lambda+\beta\right)}}-\frac{\left(\alpha_{0} \lambda+\beta\right) \lambda \alpha_{1}}{\alpha_{1} \lambda+\beta} .
$$

So that we have that the optimal maintenance rate $\gamma^{*}$ minimizing the expected cost of the system per unit time is as follows.

$$
\gamma^{*}=\left\{\begin{array}{lll}
\underline{\gamma} & \text { if } & \gamma^{\prime}<\underline{\gamma} \\
\gamma^{\prime} & \text { if } & \underline{\gamma} \leq \gamma^{\prime} \leq \bar{\gamma} \\
\bar{\gamma} & \text { if } & \gamma^{\prime}>\bar{\gamma}
\end{array}\right.
$$

This completes the proof.

\section{A.3. Proof of Lemma 5.1}

From optimality equation $v(i, j)+g_{d}=T_{m} v(i, j)$ and the definition of the operator $T_{m}$, we have that for state $(i, 2)$,

$$
v(i, 2)+g_{d}=\min _{\gamma \in[\underline{\gamma}, \bar{\gamma}]}\{\gamma v(i, 0)+(1-\gamma) v(i, 2)+c \gamma\}+i h,
$$

and for state $(i+1,2)$,

$$
v(i+1,2)+g_{d}=\min _{\gamma \in[\underline{\gamma}, \bar{\gamma}]}\{\gamma v(i+1,0)+(1-\gamma) v(i+1,2)+c \gamma\}+(i+1) h .
$$


Assume that $\gamma$ is the optimal maintenance rate for the state $(i+1,2)$. It follows from above two equations that

$$
\begin{aligned}
v(i+ & 1,2)-v(i, 2) \\
& =\min _{\gamma \in[\underline{\gamma}, \bar{\gamma}]}\{\gamma v(i+1,0)+(1-\gamma) v(i+1,2)+c \gamma\}+h-\min _{\gamma \in[\underline{\underline{\gamma}, \bar{\gamma}]}}\{\gamma v(i, 0)+(1-\gamma) v(i, 2)+c \gamma\} \\
& \geq \gamma v(i+1,0)+(1-\gamma) v(i+1,2)+c \gamma+h-\{\gamma v(i, 0)+(1-\gamma) v(i, 2)+c \gamma\} \\
& =\gamma[v(i+1,0)-v(i, 0)]+(1-\gamma)[v(i+1,2)-v(i, 2)]+h,
\end{aligned}
$$

which implies that

$$
[v(i+1,2)-v(i, 2)]-[v(i+1,0)-v(i, 0)] \geq \frac{h}{\gamma} .
$$

For $\gamma \in[\underline{\gamma}, \bar{\gamma}]$, we have $[v(i+1,2)-v(i, 2)]-[v(i+1,0)-v(i, 0)] \geq 0$. This completes the proof.

\section{A.4. Proof of Theorem 5.2}

For any state $(i, 2), i \geq 0$, we should consider the operator

$$
T_{m} v(i, 2)=\min _{\gamma \in[\underline{\gamma}, \bar{\gamma}]}\{\gamma v(i, 0)+(1-\gamma) v(i, 2)+c \gamma\}+i h
$$

Define a function $f(\gamma)=\gamma v(i, 0)+(1-\gamma) v(i, 2)+c \gamma$, in which $\gamma \in[\underline{\gamma}, \bar{\gamma}]$. Taking its first-order of $\gamma$, we have $f^{\prime}(\gamma)=v(i, 0)-v(i, 2)+c$. So that we know that the value of the function $f^{\prime}(\gamma)$ depends on the variable $\gamma$. In addition, from Lemma 5.1, we get that $v(i, 0)-v(i, 2)$ is non-increasing function to $i$, which shows that the optimal policy is a threshold policy and guarantees the existence of the threshold $N^{*}=\min \{i: v(i, 0)-v(i, 2)+c \leq 0\}$. Moreover, when the server is on the maintenance state, it contains that it is optimal to take the maintenance rate $\underline{\gamma}$ if $i<N^{*}$ otherwise $\bar{\gamma}$.

Acknowledgements. This work is partially supported by the Sun Yat-sen University Youth Teacher Cultivation Project (No. 19wkpy15), the China Postdoctoral Science Foundation (No. 2019M663197) and the National Natural Science Foundation of China (No. 11671404). The authors also gratefully acknowledge the helpful comments and suggestions of the editor and reviewers, which have improved the presentation.

\section{REFERENCES}

[1] S.K. Abeygunawardane, P. Jirutitijaroen and H. Xu, Adaptive maintenance policies for aging devices using a Markov decision process. IEEE Trans. Power Syst. 28 (2013) 3194-3203.

[2] J.S. Borrero and R. Akhavan-Tabatabaei, Time and inventory dependent optimal maintenance policies for single machine workstations: an MDP approach. Eur. J. Oper. Res. 228 (2013) 545-555.

[3] S.-P. Chen, Time value of delays in unreliable production systems with mixed uncertainties of fuzziness and randomness. Eur. J. Oper. Res. 255 (2016) 834-844.

[4] D. Chen and K.S. Trivedi, Optimization for condition-based maintenance with semi-Markov decision process. Reliab. Eng. Sys. Saf. 90 (2005) 25-29.

[5] S. Creemers and M. Lambrecht, Queueing models for appointment-driven systems. Ann. Oper. Res. 178 (2010) $155-172$.

[6] S. Gao, J. Wang and T. Van Do, Analysis of a discrete-time repairable queue with disasters and working breakdowns. RAIRO:OR 53 (2019) 1197-1216.

[7] J. Kenne and L. Nkeungoue, Simultaneous control of production, preventive and corrective maintenance rates of a failure-prone manufacturing system. Appl. Numer. Math. 58 (2008) 180-194.

[8] J.P. Kenné, E. Boukas and A. Gharbi, Control of production and corrective maintenance rates in a multiple-machine, multipleproduct manufacturing system. Math. Comput. Model. 38 (2003) 351-365.

[9] S. Khalili, H. Hosseini Nasab and F. Moobed, Optimal assignment of human resources for maintenance departments using fuzzy queuing systems. Int. J. Prod. Res. 53 (2015) 4583-4593.

[10] M.J. Kim and V. Makis, Optimal maintenance policy for a multi-state deteriorating system with two types of failures under general repair. Comput. Ind. Eng. 57 (2009) 298-303.

[11] G. Koole, Monotonicity in Markov Reward and Decision Chains: Theory and Applications. Foundations and Trends® in Stochastic Systems 1 (1) (2007) 1-76. 
[12] M.A. Lariviere and J.A. Van Mieghem, Strategically seeking service: how competition can generate Poisson arrivals. Manuf. Serv. Oper. Manage. 6 (2004) 23-40.

[13] G. Latouche and V. Ramaswami, Introduction to Matrix Analytic Methods in Stochastic Modeling. SIAM 5 (1999).

[14] A.N. Njike, R. Pellerin and J.P. Kenne, Simultaneous control of maintenance and production rates of a manufacturing system with defective products. J. Intell. Manuf. 23 (2012) 323-332.

[15] M.L. Puterman, Markov Decision Processes: Discrete Stochastic Dynamic Programming. John Wiley \& Sons Inc., New York, NY (2014).

[16] N. Salari and V. Makis, Optimal preventive and opportunistic maintenance policy for a two-unit system. Int. J. Adv. Manuf. Technol. 89 (2017) 665-673.

[17] S. Taleb and A. Aissani, Preventive maintenance in an unreliable M/G/1 retrial queue with persistent and impatient customers. Ann. Oper. Res. 247 (2016) 291-317.

[18] H.C. Tijms, Stochastic Models: An Algorithmic Approach. In Vol. 303 of Probability and Statistics - Applied Probability and Statistics Section. John Wiley \& Sons Inc., New York, NY (1994).

[19] K.-H. Wang, W.-L. Chen and D.-Y. Yang, Optimal management of the machine repair problem with working vacation: Newton's method. J. Comput. Appl. Math. 233 (2009) 449-458.

[20] C.-H. Wu, W.-C. Lee, J.-C. Ke and T.-H. Liu, Optimization analysis of an unreliable multi-server queue with a controllable repair policy. Comput. Oper. Res. 49 (2014) 83-96.

[21] R.H. Yeh, K.-C. Kao and W. L. Chang, Optimal preventive maintenance policy for leased equipment using failure rate reduction. Comput. Ind. Eng. 57 (2009) 304-309.

[22] G. Yingkui and L. Jing, Multi-state system reliability: a new and systematic review. Proc. Eng. 29 (2012) $531-536$.

[23] Z. Zheng, W. Zhou, Y. Zheng and Y. Wu, Optimal maintenance policy for a system with preventive repair and two types of failures. Comput. Ind. Eng. 98 (2016) 102-112. 